\title{
Middle-molecular-weight Glycoclusters for the Crosslinking of Multivalent Lectins
}

\author{
Makoto Ogata \\ Faculty of Food and Agricultural Sciences, Fukushima University, Fukushima, Fukushima 960-1296, Japan \\ TEL: +81-24-503-4982, E-mail: ogata@agri.fukushima-u.ac.jp \\ (Received on March 9, 2021, accepted on March 29, 2021)
}

Key Words: glycocluster, lectin, cross-link, agglutination, molecular recognition

\begin{abstract}
Multivalent ligands with a regular arrangement of carbohydrates, such as natural glycoproteins, glycopeptides, and branched oligosaccharides, play an important role as in vivo molecular recognition elements for lectins. In recent years, attempts have been actively made to develop functional glyco-materials, such as cell culture substrates and virus adsorbents, by artificially imitating and reproducing the molecular recognition ability of natural multivalent glycans. Multivalent lectins with two or more sugar-binding sites bind to multivalent ligands in a structure-specific manner and are thereby crosslinked to form an aggregate. Although this crosslinking reaction is a universal phenomenon, many unclear points remain in the series of mechanisms involved in the formation of cross-linked lectin complexes. Furthermore, multivalent lectins are also present on the surface of pathogenic bacteria and pathogenic viruses, and may be used as molecular targets in the development of toxin protein neutralizers and antiviral agents. To address these issues, middle-molecular-weight glycoclusters with a well-defined structure and a controlled number of sugar chain clusters have been designed and synthesized as potential cross-linking agents for multivalent lectins. This review provides an overview of the synthesis and utilization of middle-molecular-weight glycoclusters.
\end{abstract}

\section{A. Introduction}

Lectins are sugar-binding proteins found in a wide variety of animals, plants, microorganisms, and viruses (1). Common structural features of many lectins include oligomerization and multivalent sugar-binding activity (2). These structural properties are clear from three-dimensional structural information derived from X-ray crystal structure analysis of lectins $(3,4)$. Carbohydrates present both on cell surfaces and as free substances function as ligands for lectins in the living body, and thus play important roles in biological defense mechanisms such as signal transduction between cells and immunity (1). In reality, multivalent lectins acquire high binding affinity through a plurality of interactions (so-called "glycocluster effects") with glycoconjugates containing multivalent carbohydrates $(5,6)$. Furthermore, multivalent lectins can form a crosslinked complex with multivalent carbohydrates that have an appropriate molecular structure such as a glycopeptide (7). While research on the ability of multivalent lectins to form crosslinks with multivalent carbohydrates dates back many years to the $1900 \mathrm{~s}$ (8), the nano-to-submicron scale morphological changes that accompany the cross-linking of these multivalent lectins are currently very interesting to the field of modern carbohydrate science.

Galactose (Gal)-specific animal lectins (galactins) are one of the most studied lectins in terms of their cross-linking activity (7). For example, galectin-1 and -2 exist in a dimer, and one sugar-binding site is located at each end of the dimer. Due to this structural property, such polyvalent lectins can form cross-linked complexes with multivalent carbohydrates and glycoconjugates, and are involved in binding to the cell surface and controlling the distance between glycoproteins (7). For example, it is known that cross-linking of cell surface carbohydrates by galectin is involved in the mitogenic activities, and cross-linking of glycoproteins by galectin-1 induces apoptosis in activated human T-cells $(9,10)$. In addition to galectins, many other multivalent lectins can crosslink with multivalent carbohydrates such as glycoproteins, glycopeptides, and branched oligosaccharides, and it is understood that this is a universal phenomenon $(11,12)$.

Against this background, glyco-clustering technology, in which lectin-recognized carbohydrates are multivalently arranged on polymers and particles, has been developing since the $1990 \mathrm{~s}$ $(6,13-16)$. Via this technology, it is possible to dramatically increase the binding affinity of lectins for originally weak-binding carbohydrates. This process is called the glycocluster effect. In recent years, various artificial multivalent glyco-materials (e.g., glyco-polymers, glyco-particles, and glyco-thin films) have been developed and widely used as a tool for elucidating the lectincarbohydrate interaction (14, 17-23). Furthermore, because these multivalent glyco-materials also have the property of strongly binding to, for example, viruses, microorganisms, and toxin proteins that possess lectins, they are also used as adsorbents or detection probes for these factors (24-31).

In addition to the above-mentioned macromolecules (i.e., glyco-polymers and glyco-particles), attempts have been made to 
bind about 2-20 glycans to the backbone of middle-molecularweight species (e.g., dendrimers, calixarene, cyclic oligosaccharides, and branched peptides) (32-37). Although the binding affinity of such middle-molecular-weight glycoclusters for lectins is inferior to that of the macromolecules such as glyco-polymers, it is expected that these glycoclusters may be developed as anti-pathogenic agents based on their structural clarity $(38,39)$. More interestingly, by proper molecular design of these middle-molecular-weight glycoclusters, the reaction of cross-linked complex formation with multivalent lectins in vitro can be reproduced (40). In this review, we focus on multivalent lectin cross-linking using middle-molecular-weight glycoclusters, and describe cross-linking of lectins, thermodynamic analysis, and removal of the pathogenic viruses by cross-linking.

\section{B. Tetravalent LacNAc-glycoclusters as Cross-linkers of Erythrina cristagalli Agglutinin}

The most common strategy for synthesizing multivalent lectin crosslinkers is to increase the number of carbohydrates per molecule to $2,3,4$, and so on $(32,38,39)$. As a result of the glycocluster effect, high binding affinity and cross-linking to the target multivalent lectin can be obtained. So far, various middle-molecular-weight glycoclusters with affinity for plant lectins (e.g., concanavalin A), animal lectins (e.g., galectins), and pathogen lectins (e.g., LecA from Pseudomonas aeruginosa), have been developed by this approach $(32,39,41)$. We have also reported the synthesis of middle-molecular-weight glycoclusters that mimic the $\mathrm{N}$ glycans of natural glycoproteins $(42,43)$. These compounds have 2-4 chitobiose structures in one molecule. Furthermore, by analyzing the interaction between these multivalent ligands and Triticum vulgaris agglutinin (WGA), a plant multivalent lectin, it has been demonstrated that higher numbers of carbohydrates per molecule lead to a dramatic increase in binding affinity. In addition, these multivalent ligands have been shown to undergo a cross-linking reaction with WGA to form aggregates (43).

For some multivalent lectins, however, binding affinity and cross-linking ability may not be improved by simply increasing the number of glycans in the ligand. For example, Erythrina cristagalli agglutinin (ECA) isolated from the seeds of E. cristagalli can be classified as a lectin that is less prone to the glycocluster effect $(43,44)$. This plant lectin has binding affinity for carbohydrates containing $\mathrm{Gal}$ or $\mathrm{N}$-acetylgalactosamine at the non-reducing end. It is a homodimer composed of a monomer with a $\beta$-sandwich structure, and it has sugar-binding sites at both ends (45). Due to the structural feature of the long distance between the two sugarbinding sites, the glycocluster effect observed for many multivalent lectins is much smaller for ECA $(43,46)$. Furthermore, many of the middle-molecular-weight glycoclusters reported so far are de- signed so that all sugar chains are utilized for lectin binding, resulting in cross-linking ability and improved affinity. Although such a design is expected to increase the enthalpy involved in binding affinity, however, it also results in a higher entropy penalty. Against this background, in recent years we have synthesized the tetravalent $\mathrm{N}$-acetyllactosamine (LacNAc)-cluster, which has four LacNAc structures in one molecule (47). This glycocluster was molecularly designed to have high affinity and ability to form crosslinks with ECA. Here, we describe synthesis of this novel ECA crosslinking agent, which has a reduced entropy penalty.

First, three types of tetravalent double-headed glycoclusters [(LacNAc $)_{4}$-DHGs] with four LacNAc residues in one molecule and different skeletal lengths were designed and synthesized as ECA cross-linking agents. Specifically, (LacNAc) $)_{4}-\mathrm{DHG}_{-} \mathrm{C}_{6}(\mathbf{1})$, $(\mathrm{LacNAc})_{4}-\mathrm{DHG}_{-\mathrm{C}_{12}}$ (2) and (LacNAc) $)_{4}-\mathrm{DHG}-\mathrm{C}_{18}$ (3) were obtained by introducing two bivalent LacNAc glycosides into both terminal carboxy groups of three types of dicarboxylic acid skeletons with different alkyl chain lengths $\left[\mathrm{HOOC}-\left(\mathrm{CH}_{2}\right)_{\mathrm{n}}-\mathrm{COOH}\right.$; $n=4,10,16$ ] (Fig. 1). As control compounds, (LacNAc) -EGTAs $^{-E}$ (4 and 5), in which four LacNAc glycosides were introduced into ethylene glycol tetraacetic acid (EGTA), and bivalent LacNAcglycoclusters (6 and 7) were used (Fig. 1). Compounds 4 and 5 were designed as tetravalent LacNAc-clusters in which the distances between the four LacNAc residues were evenly extended. Next, various interactions between these ligands and ECA were analyzed to evaluate the effect of the different skeleton structures of ( $\mathrm{LacNAc})_{4}$-DHGs on their ability to bind ECA.

Isothermal titration calorimetry (ITC) analysis showed that the binding of all sugar chain ligands to ECA was enthalpy-driven (Table 1). In addition, the stoichiometric ratio of the reaction showed that the binding ratio of all three (LacNAc) $)_{4}$-DHGs to ECA was $1: 2$ (Table 1). On the one hand, the value of $\Delta \mathrm{H}$ for the bond between compound $\mathbf{3}$ with the longest skeletal alkyl chain length and ECA was $-34.6 \mathrm{kcal} / \mathrm{mol}$, which was the lowest value among the tetravalent glycoclusters (1-5) (Table 1). On the other hand, the entropy penalty $(-\mathrm{T} \Delta \mathrm{S}=27.1 \mathrm{kcal} / \mathrm{mol})$ for the binding of compound 3 to ECA was the lowest as compared with the other tetravalent glycoclusters; as a result, compound $\mathbf{3}$ showed the strongest binding affinity $\left(K_{\mathrm{d}}=2.5 \mu \mathrm{M}\right)$ for ECA (Table 1$)$. The binding affinity of compound 3 was 14 -fold stronger than that of the monovalent LacNAc glycoside (8); moreover, binding affinity increased as the alkyl chain length of the (LacNAc) $)_{4}$-DHGs skeleton was extended (Table 1). These results are in good agreement with molecular dynamics simulations with explicit water molecules for compounds $\mathbf{1}, 2$ and 3 (47). In particular, for the compounds 1 and 2, their own LacNAc residues in the same arm bound strongly with each other, thus stabilizing the structures of $\mathbf{1}$ and $\mathbf{2}$. In contrast, compound 

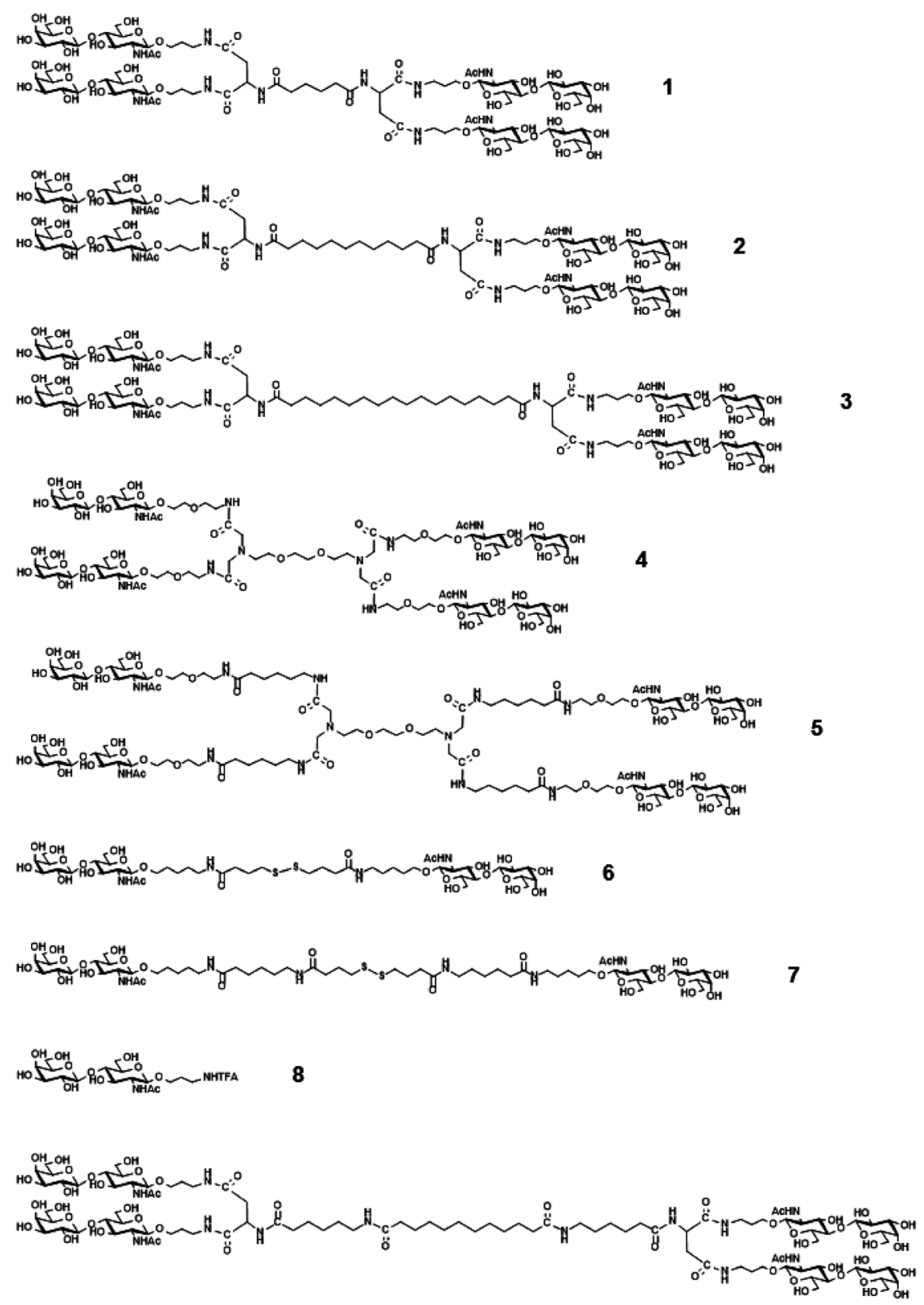

Fig. 1. Structures of tetravalent LacNAc-glycoclusters 1-5 and 9, bivalent LacNAc-glycoclusters 6 and 7, and monovalent LacNAc-glycoside 8.

3, which had the longest skeletal alkyl chain length, did not show packing between its LacNAc residues. That is, flexible compound 3, which has the closely-situated two LacNAc units in the same arm, was suitable for binding to ECA. In addition, no increase in binding affinity for ECA was observed for molecular designs such

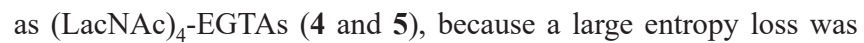
observed due to an increase in the number of lectins bound to the tetravalent glycoclusters (Table 1). Based on these observations, (LacNAc) $)_{4}-\mathrm{DHG}_{24}$ (9), which has a longer skeleton than 3, was additionally synthesized, but its binding affinity was almost the same as that of $\mathbf{3}$ (Fig. 1). Furthermore, compounds $\mathbf{3}$ and $\mathbf{9}$ showed maximum precipitation when mixed with ECA in a molar ratio of
$1: 2$, yielding a particle size of $2 \mu \mathrm{m}$ and demonstrating the ability to form a large ligand-ECA crosslinked complex. Collectively, these results show that, in addition to the glycocluster effect, reduction of entropy penalty due to modification of the skeletal structure is important for the development of middle-molecular-weight glycoclusters with high affinity for ECA (47).

\section{Tetravalent Sialo-glycoclusters as Cross-linkers of Sambucus sieboldiana Agglutinin}

In recent years, middle-molecular-weight glycoclusters have been widely used not only as cross-linking agents for animal and plant lectins, but also as tools for elucidating biological phenom- 
ena related to sugar chains and as inhibitors against pathogens (38, 39). To further expand the scope of this research, however, there is a problem in the synthesis of middle-molecular-weight glycoclusters that must be overcome. The problem is that most traditional middle-molecular-weight glycoclusters are limited to compounds in which monosaccharides or disaccharides are bound to a skeletal structure $(26,38,48)$. This not only reduces the solubility of the ligands, but also limits the lectins that can be targeted. Therefore, we developed a new middle-molecular-weight glycocluster for Sambucus sieboldiana agglutinin (SSA), which has a wide sugar-

Table 1. Multivalent compounds targeted to ECA lectin.

\begin{tabular}{ccccccr}
\hline & & & \multicolumn{5}{c}{ ITC } \\
\cline { 4 - 7 } Compounds & Valency & $\begin{array}{c}\mathrm{MIC}^{\mathrm{a}} \\
(\mathrm{LacNAc} \mu \mathrm{M})^{\mathrm{b}}\end{array}$ & $n^{\mathrm{c}}$ & $\begin{array}{c}\Delta H \\
\left(\mathrm{kcal} \mathrm{mol}^{-1}\right)\end{array}$ & $\begin{array}{c}-T S \\
\left(\mathrm{kcal} \mathrm{mol}^{-1}\right)\end{array}$ & $\begin{array}{c}K_{\mathrm{d}} \\
(\mu \mathrm{M})\end{array}$ \\
\hline $\mathbf{1}$ & 4 & 6 & 0.48 & -39.7 & 32.8 & 7.2 \\
$\mathbf{2}$ & 4 & 3 & 0.47 & -38.4 & 31.1 & 3.9 \\
$\mathbf{3}$ & 4 & 0.76 & 0.46 & -34.6 & 27.1 & 2.5 \\
$\mathbf{4}$ & 4 & 12 & 0.51 & -35.1 & 28.2 & 7.2 \\
$\mathbf{5}$ & 4 & 6 & 0.37 & -51.1 & 44.0 & 6.0 \\
$\mathbf{6}$ & 2 & 3 & 1.02 & -21.7 & 15.3 & 17.5 \\
$\mathbf{7}$ & 2 & 3 & 0.69 & -27.6 & 21.2 & 15.6 \\
$\mathbf{8}$ & 1 & - & 0.99 & -25.3 & 19.4 & 35.2 \\
$\mathbf{9}$ & 4 & 0.56 & 0.50 & -29.2 & 21.6 & 2.3 \\
\hline
\end{tabular}

${ }^{a}$ Minimum concentration required to completely inhibit hemagglutination.

${ }^{\mathrm{b}}$ Concentration of LacNAc units.

${ }^{\mathrm{c}}$ Binding stoichiometry was defined as the number of ligands bound per monomer of ECA. binding site that can interact with multiple sugar residues in the sugar chain (49). SSA is a plant lectin present in the bark of $S$. sieboldiana, and is a heterotetrameric glycoprotein with two sugarbinding sites (50-52). In addition, SSA shows binding affinity for sugar chains containing Neu5Ac $\alpha 2,6 \mathrm{Gal}$ and Neu5Ac $\alpha 2,6 \mathrm{GalNAc}$ (50-52). In an attempt to produce multivalent ligands for SSA, middle-molecular-weight glycoclusters with four trisaccharide- to pentasaccharide-glycosides in one molecule were synthesized by using a chemoenzymatic approach. Here, we describe a useful SSA cross-linking agent that we have developed by controlling the valency and the spatial arrangement of ligand sugar chains.

First, LacNAc glycoside with a trifluoroacetamide group at the end of the aglycone moiety was synthesized by a condensation reaction using endoglucanase I obtained from cellulase from Trichoderma reesei $(53,54)$. Next, a glycan elongation reaction using two glycosyltransferases $(\beta$-1,4-galactosyltransferase and $\beta-1,3-N$-acetylglucosaminyltransferase) was carried out to synthesize $\operatorname{LacNAc} \beta 1,3 \mathrm{LacNAc}$ glycosides from LacNAcglycoside. After deprotecting the aglycone terminal, the resulting di- and tetra-glycosides were then introduced into EGTA to synthesize two tetravalent asialo-glycoclusters. Last, regioselective sialylation of the tetravalent asialo-glycoclusters using $\alpha$-2,6-sialylltransferase was performed to synthesize [Neu5Ac $\left.\alpha 2,6 \mathrm{LacNAc}-\beta-\mathrm{O}\left(\mathrm{CH}_{2}\right)_{2} \mathrm{O}\left(\mathrm{CH}_{2}\right)_{2} \mathrm{NH}-\right]_{4}$-EGTA (10) and [Neu $\left.5 \mathrm{Ac} \alpha 2,6 \mathrm{LacNAc} \beta 1,3 \mathrm{LacNAc}-\beta-\mathrm{O}\left(\mathrm{CH}_{2}\right)_{2} \mathrm{O}\left(\mathrm{CH}_{2}\right)_{2} \mathrm{NH}-\right]_{4}-$ EGTA (11) (49) (Fig. 2). Separately, [Neu5Ac $\alpha 2,6$ LacNAc- $\beta$ -

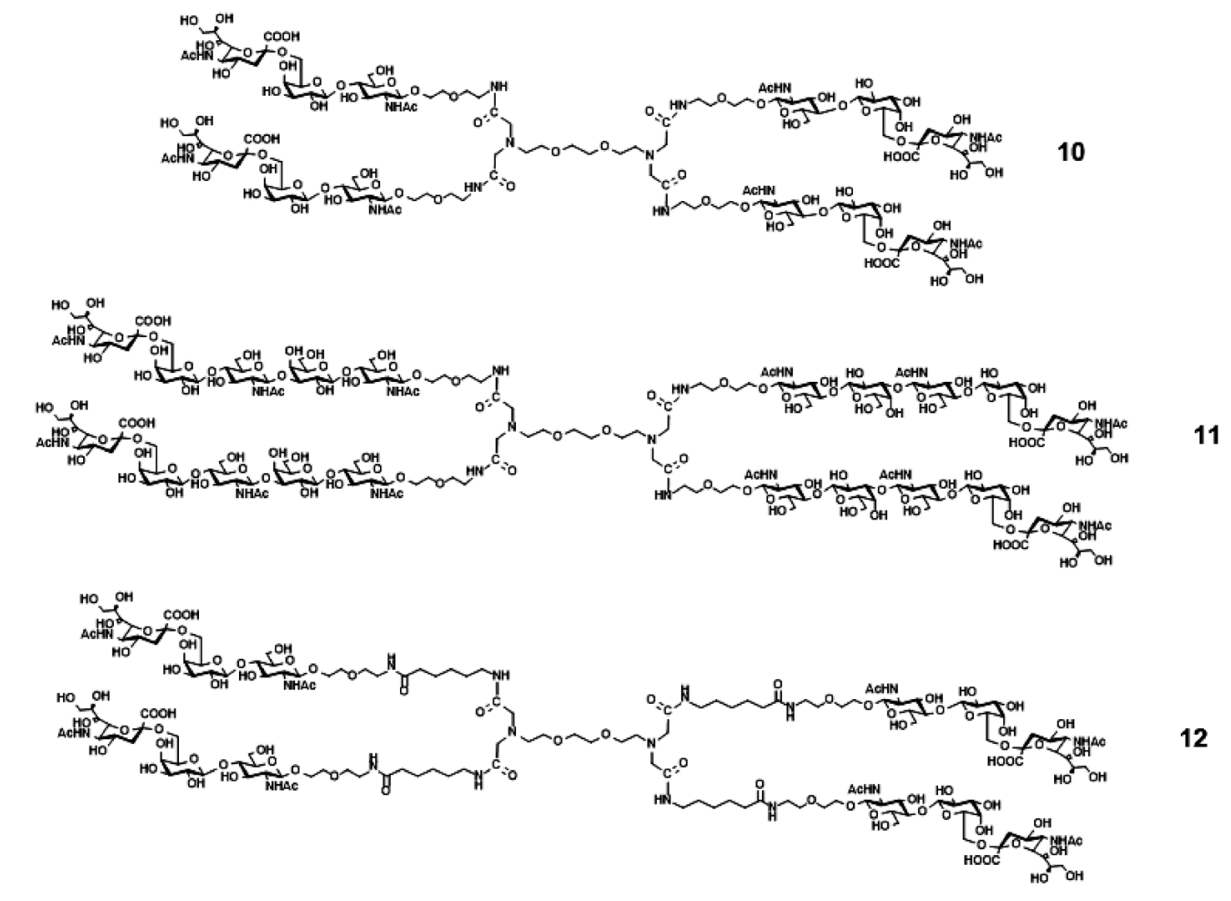

Fig. 2. Structures of tetravalent sialo-glycoclusters 10, 11 and 12 . 


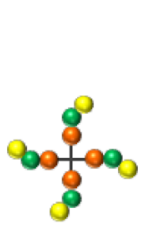

10

\begin{tabular}{cccc}
\hline \multicolumn{4}{c}{ ITC } \\
\hline$n$ & $\begin{array}{c}\Delta H \\
\left(\mathrm{kcal} \mathrm{mol}^{-1}\right)\end{array}$ & $\begin{array}{c}-T \Delta S \\
\left(\mathrm{kcal} \mathrm{mol}^{-1}\right)\end{array}$ & $\begin{array}{c}K_{\mathrm{d}} \\
(\mathrm{nM})\end{array}$ \\
\hline 0.47 & -57.9 & 48.3 & 99 \\
\hline
\end{tabular}

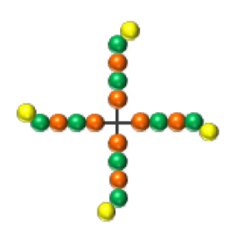

11
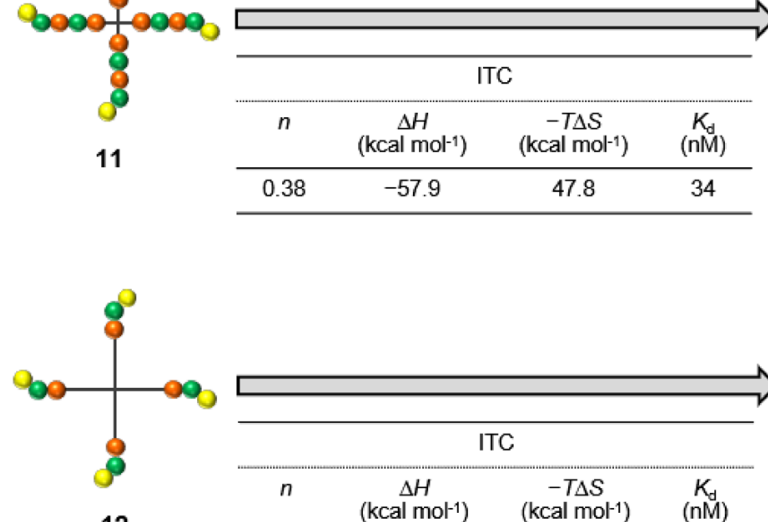

12
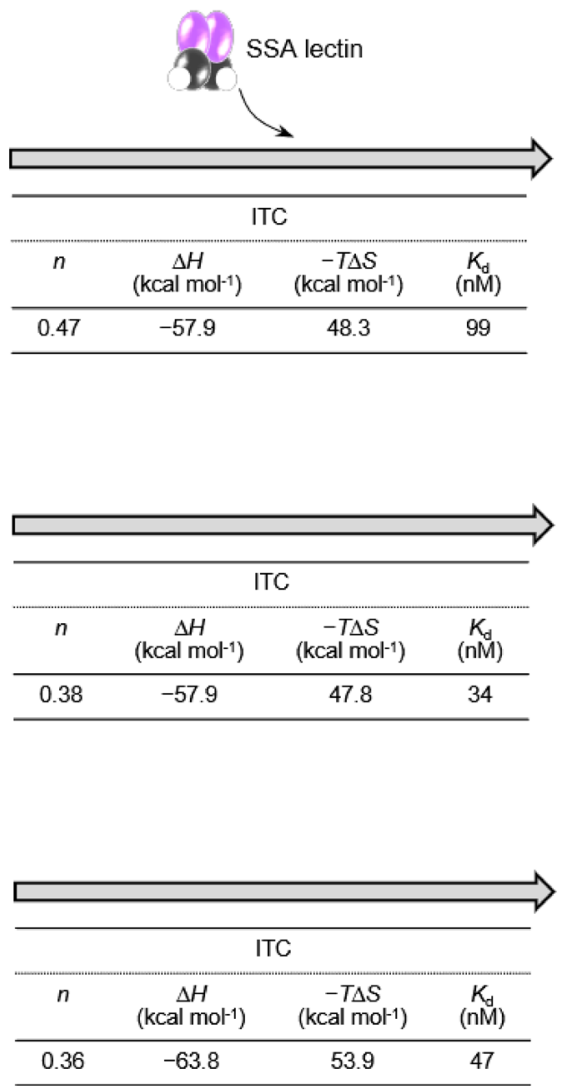

$10: \operatorname{SSA}=1: 2$

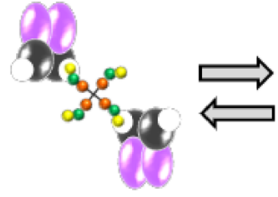

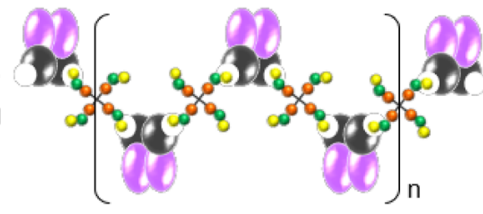

$10: \mathrm{SSA} \approx 1: 1$

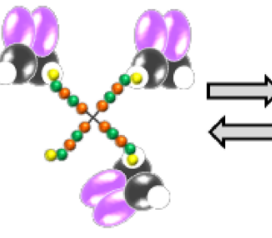

$11:$ SSA $=1: 3$

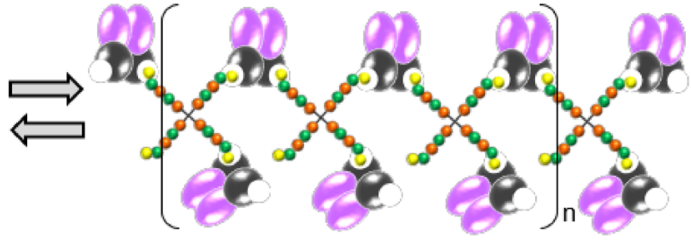

$11: \mathrm{SSA} \approx 1: 2$

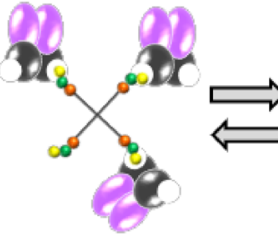

$12: \operatorname{SSA}=1: 3$

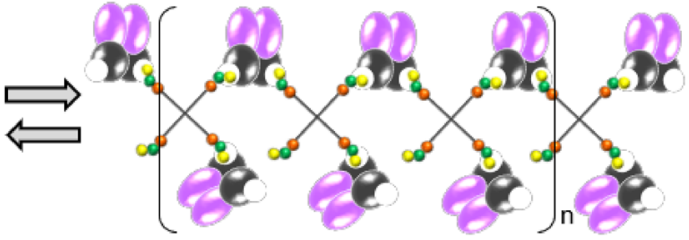

$12: \mathrm{SSA} \approx 1: 2$

Fig. 3. Schematics showing the crosslinking of SSA lectin with tetravalent sialo-glycoclusters.

$\left.\mathrm{O}\left(\mathrm{CH}_{2}\right)_{2} \mathrm{O}\left(\mathrm{CH}_{2}\right)_{2} \mathrm{NHCO}\left(\mathrm{CH}_{2}\right)_{5} \mathrm{NH}-\right]_{4}$-EGTA (12), in which the length corresponding to one repetition of LacNAc of $\mathbf{1 1}$ was replaced with an alkyl spacer (49), was also synthesized (Fig. 2). Subsequently, the binding between these tetravalent sialoglycoclusters and SSA was evaluated by ITC. As a result, compound 11 showed the lowest dissociation constant $\left(K_{\mathrm{d}}=34 \mathrm{nM}\right)$, indicating a binding affinity that was 28 -fold stronger than that of the monovalent Neu5Ac $\alpha 2,6 \operatorname{LacNAc}\left(K_{\mathrm{d}}=940 \mathrm{nM}\right)$ (Fig. 3). Interestingly, the number of SSA bonds to one glycocluster molecule differed between compound $\mathbf{1 0}$ and compounds $\mathbf{1 1}$ and 12: that is, two molecules of SSA bound to one molecule of $\mathbf{1 0}$, whereas three molecules of SSA bound to one molecule of 11 or $\mathbf{1 2}$ (Fig. 3). The morphological changes associated with the cross-linking of these ligands were also evaluated by quantitative precipitation assay and dynamic light scattering (DLS), which demonstrated that the crosslinking aggregates formed in the concentration range indicated by the ITC results. As a result, compound $\mathbf{1 1}$ showed maximum precipitation at a mixing ratio with SSA of $1: 2$, and the particle size of the crosslinked complex was about 30 -fold larger than that of the SSA monomer (22.5 nm) (Fig. 3). Overall, we succeeded in synthesizing various middle-molecular-weight glycoclusters in which long-sugar chains were bound by using this chemoenzymat- ic approach. By analyzing various interactions between the synthetic ligand library and SSA (49), we can propose a mechanism for cross-linked complex formation (Fig. 3).

\section{Crosslinking of Human Polyomaviruses by Tetra- valent Sialo-glycoclusters}

Lectins are also present on the surface of pathogenic viruses involved in various infectious diseases, and establish an infection by binding to sugar chains on the surface of host cells (1). When comparing the pathogenic virus human polyomavirus and SSA, for example, there are differences of about 2-fold in particle size and about 200-fold in the number of sugar-binding sites $(49,55$, 56). So, how do middle-molecular-weight glycoclusters act on such huge multivalent lectins (virus particles)? In response to this question, a large number of middle-molecular-weight glycoclusters with high binding affinity for pathogenic viruses have been designed, and research on their utility as antiviral agents is in progress (38). By contrast, there are very few studies on the formation of cross-linked complexes between middle-molecular-weight glycoclusters and pathogenic viruses (57). Therefore, we investigated the agglutination reaction of pathogenic human polyomavirus using SSA crosslinkers (tetravalent sialo-glycoclusters). 

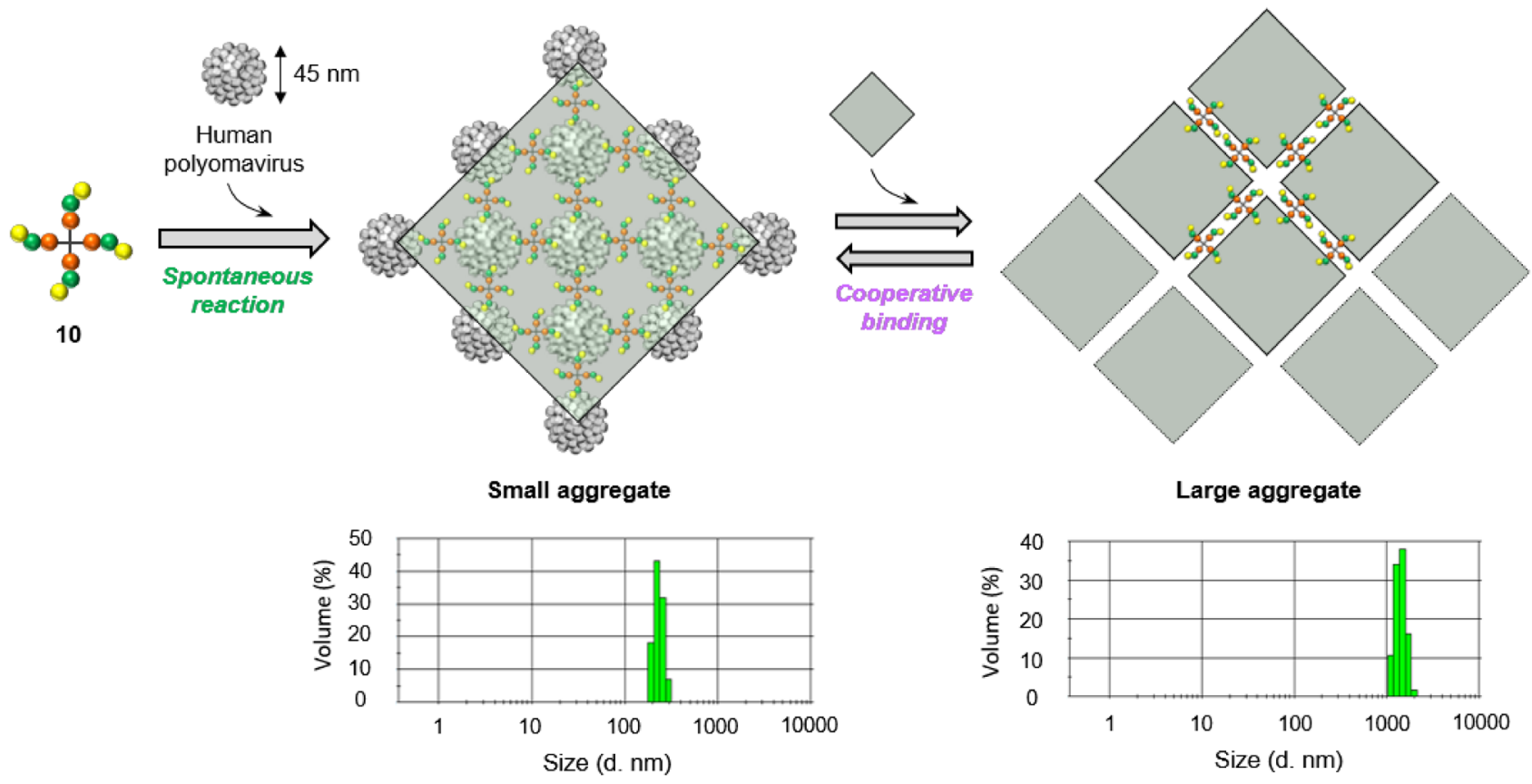

Fig. 4. Schematic showing the crosslinking of human polyomavirus with tetravalent sialo-glycoclusters.

The human polyomavirus targeted in the study, Merkel cell polyomavirus (MCPyV), consists of 72 capsomers, which mainly comprise a major capsid viral protein (VP1) with binding sites for sialylated glycans $(58,59)$. We determined conditions for forming crosslinked viral aggregates with good reproducibility by examining various mixing ratios of tetravalent sialo-glycoclusters (10 and 12) and virus-like particles of MCPyV (MCPyV-LP) (60). We evaluated the detailed binding process between the tetravalent sialo-glycoclusters and MCPyV-LP by DLS, which indicated that tetravalent sialo-glycoclusters formed a crosslinked complex by multivalent binding with VP1 on the surface of MCPyV-LP. The size of the aggregates increased with the amount of added tetravalent sialo-glycoclusters until a maximum value was reached. Specifically, MCPyV-LP, which has a particle size of about $45 \mathrm{~nm}$, was cross-linked with tetravalent sialo-glycoclusters, resulting in a complex with an average particle size of about $2 \mu \mathrm{m}$ (i.e., $\sim 40$-fold larger) (Fig. 4). In the comparison of ligands, compound $\mathbf{1 0}$ with a short spacer showed stronger binding affinity than $\mathbf{1 2}$ with a long spacer. Interestingly, DLS measurements showed that this series of agglutination reactions proceeded in two stages: in the first stage, small aggregates containing crosslinked ligand-viral complexes were spontaneously formed (Fig. 4); in the next stage, there was gradual growth of the aggregates due to coordinated binding between the small aggregates generated in the initial stage (Fig. 4). Observation of these aggregates with a transmission electron microscope (TEM) showed that the virus particles became dense due to cross-linking via the ligand. These results mean that even a large multivalent lectin such as a virus particle can form a microscale complex by a structure-specific cross-linking reaction with a middle-molecular-weight glycoclusters.

We also carried out a membrane filtration assay of MCPyVLP, inspired by the morphological changes of the virus particles caused by crosslinking with the above-mentioned middlemolecular-weight glycoclusters. As a result, the virus particles were retained on the filtration membrane when the compound 10-MCPyV-LP aggregates were applied to a general sterilization membrane filter $(0.20 \mu \mathrm{m})$ for microbial filtration. Furthermore, this membrane filtration assay showed effective virus trapping of infectious human polyomavirus JCPyV (60). In other words, these results suggest the possibility of virus aggregation using middlemolecular-weight glycoclusters as a new method for virus capture and/or purification method.

\section{E. Conclusions}

In this review, we have outlined the synthesis of novel middle-molecular-weight glycoclusters and in vitro experiments on the cross-linking reactions of multivalent lectins with these ligands. By controlling the three-dimensional arrangement of ligands via the molecular design of middle-molecular-weight glycoclusters, it has been possible to obtain molecules with both high binding affinity for multivalent lectins and the ability to form crosslinked complexes. At present, however, the evaluation of cross-linking between middle-molecular-weight glycoclusters and multivalent lectins is limited to observing the state before and after formation of the 
cross-linked complex, and it is difficult to analyze the cross-linking reaction in real time. In the future, it is expected that, by coupling our syntheses with the latest measurement systems that can evaluate the entire cross-linking reaction of multivalent lectins, it will be possible to elucidate the individual steps of the cross-linking reaction and establish a methodology for the creation of new ligands.

\section{Acknowledgments}

This work was supported by JSPS KAKENHI grant numbers 24778011, 17K05024, and 20K05855.

\section{References}

1. Varki, A. (1993) Glycobiology 3, 97-130.

2. Lis, H., and Sharon, N. (1998) Chem. Rev. 98, 637-674.

3. Vijayan, M., and Chandra, N. (1999) Curr. Opin. Struct. Biol. 9, 707-714.

4. Fujimoto, Z., Tateno, H., and Hirabayashi, J. (2014) Methods Mol. Biol. 1200, 579-606.

5. Lee, Y., et al. (1983) J. Biol. Chem. 258, 199-202.

6. Lundquist, J. L., and Toone, E. J. (2002) Chem. Rev. 102, 555-578.

7. Brewer, C. F. (1997) Trends Glycosci. Glycotechnol. 9, 155-165.

8. Landsteiner, K., and Raubitschek, H. (1907) Zentralbl. Bakteriol. 45, 660-667.

9. Sanford, G. L., and Harris-Hooker, S. (1990) FASEB J. 4, 29122918.

10. Perillo, N. L., et al. (1995) Nature 378, 736-739.

11. Nicolson, G. L. (1976) Biochim. Biophys. Acta 458, 1-72.

12. Dessen, A., et al. (1995) Biochemistry 34, 4933-4942.

13. Mammen, M., Choi, S.-K., and Whitesides, G. M. (1998) Angew. Chem. Int. Ed. 37, 2754-2794.

14. Kiessling, L. L., Gestwicki, J. E., and Strong, L. E. (2006) Angew. Chem. Int. Ed. 45, 2348-2368.

15. Dam, T. K., and Brewer, C. F. (2008) Biochemistry 47, 8470-8476.

16. Fasting, C., et al. (2012) Angew. Chem. Int. Ed. 51, 10472-10498.

17. Endo, T., et al. (2011) Sens. Mater. 23, 135-146.

18. Gening, M. L., et al. (2013) Chemistry 19, 9272-9285.

19. Matsuura, K. (2013) Trends Glycosci. Glycotechnol. 25, 227-239.

20. Miura, Y., Hoshino, Y., and Seto, H. (2016) Chem. Rev. 116, $1673-$ 1692.

21. de la Calle, A. C., et al. (2019) Macromol. Biosci. 19, 1900033.

22. Rahkila, J., et al. (2019) ChemBioChem 20, 203-209.
23. Yamauchi, N., et al. (2019) Colloids Surf. A Physicochem. Eng. Asp. 580, 123754.

24. Umemura, M., et al. (2008) J. Med. Chem. 51, 4496-4503.

25. Ogata, M., et al. (2009) Bioconjug. Chem. 20, 538-549.

26. Chabra, Y. M., and Roy, R. (2010) Adv. Carbohydr. Chem. Biochem. 63, 165-393.

27. Suzuki, K., et al. (2012) Bioorg. Med. Chem. 20, 446-454.

28. Tanaka, T., et al. (2014) ACS Macro Lett. 3, 1074-1078.

29. Kato, T., et al. (2015) J. Biotechnol. 209, 50-57.

30. Ogata, M., et al. (2016) Carbohydr. Polym. 153, 96-104.

31. Ogata, M., et al. (2019) ACS Appl. Bio Mater. 2, 1255-1261.

32. Roy, R. (2003) Trends Glycosci. Glycotechnol. 15, 291-310.

33. Cecioni, S., et al. (2009) Chemistry 15, 13232-13240.

34. Beckmann, H. S. G., et al. (2012) J. Org. Chem. 8, 819-826.

35. Samuel, E., et al. (2017) Chem. Rev. 117, 6538-6631.

36. Kašáková, M., et al. (2018) Carbohydr. Res. 469, 60-72.

37. Günther, S. C., et al. (2020) Sci. Rep. 10, 768.

38. Bernardi, A., et al. (2013) Chem. Soc. Rev. 42, 4709-4727.

39. Cecioni, S., Imberty, A., and Vidal, S. (2015) Chem. Rev. 115, 525561.

40. Misawa, Y., et al. (2008) J. Biochem. 143, 21-30.

41. Yang, L.-Y., et al. (2010) Carbohydr. Res. 345, 2124-2132.

42. Misawa, Y., et al. (2008) Carbohydr. Res. 343, 434-442.

43. Masaka, R., et al. (2010) Bioorg. Med. Chem. 18, 621-629.

44. Iglesias, J. L., Lis, H., and Sharon, N. (1982) Eur. J. Biochem. 123 247-252.

45. Turton, K., et al. (2004) Glycobiology 14, 923-929.

46. Cecioni, S., et al. (2012) Carbohydr. Res. 356, 132-141.

47. Ogata, M., et al. (2016) Bioorg. Med. Chem. 24, 1-11.

48. Gening, M. L., et al. (2013) Chemistry 19, 9272-9285.

49. Ogata, M., et al. (2012) Bioconjug. Chem. 23, 97-105.

50. Shibuya, N., et al. (1989) J. Biochem. 106, 1098-1103.

51. Tazaki, K., and Shibuya, N. (1989) Plant Cell Physiol. 30, 899-903.

52. Kaku, H., et al. (1996) J. Biol. Chem. 271, 1480-1485.

53. Ogata, M., et al. (2007) Bioorg. Med. Chem. 15, 1383-1393.

54. Ogata, M., et al. (2010) Carbohydr. Res. 345, 2623-2629.

55. Li, T.-C., et al. (2015) PLoS One 10, e0115646.

56. Baier, M., et al. (2019) Macromol. Biosci. 19, 1800426.

57. Glick, G. D., et al. (1991) J. Biol. Chem. 266, 23660-23669.

58. Neu, U., Bauer, J., and Stehle, T. (2011) Curr. Opin. Struct. Biol. 21, 610-618.

59. Neu, U., et al. (2012) PLoS Pathog. 8, e1002738.

60. Ogata, M., et al. (2020) ACS Omega 5, 211940-221947.

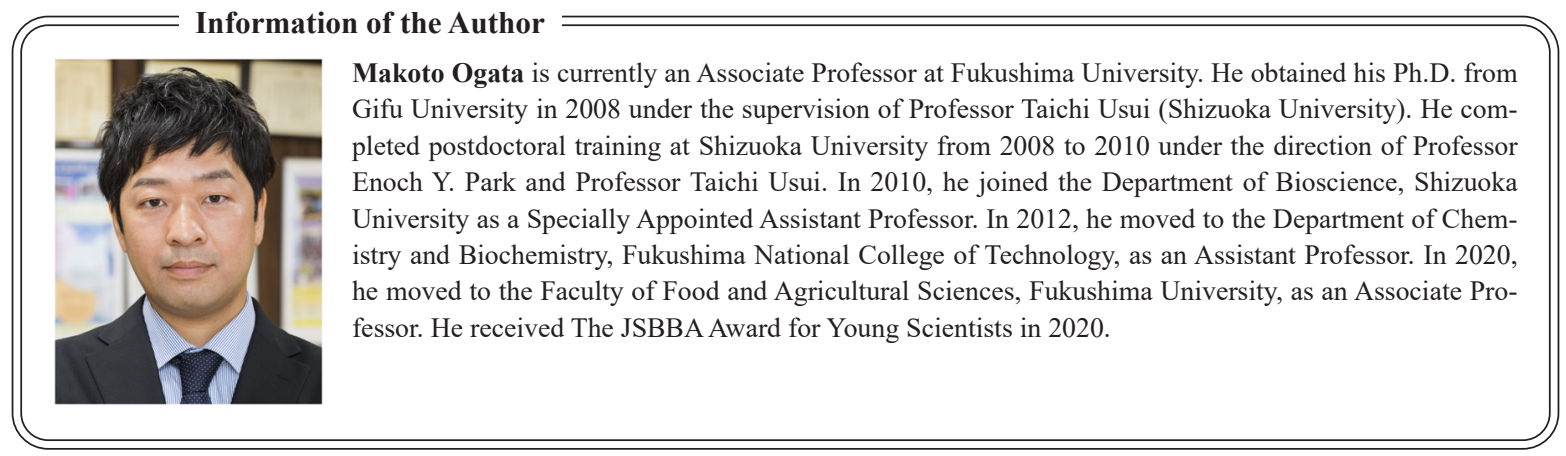

